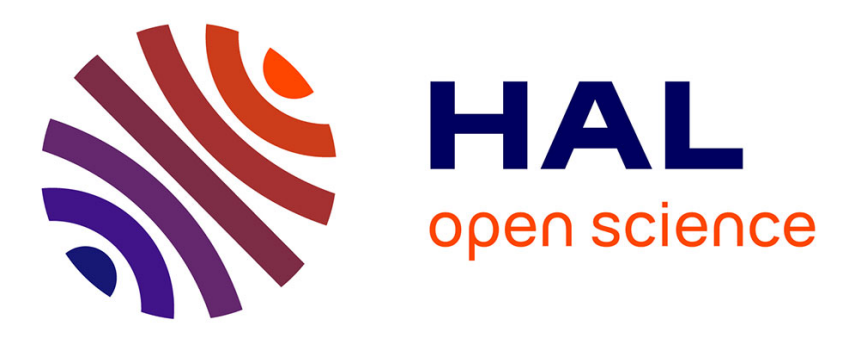

\title{
Towards Robotic MAGMaS: Multiple Aerial-Ground Manipulator Systems
}

Nicolas Staub, Mostafa Mohammadi, Davide Bicego, Domenico Prattichizzo, Antonio Franchi

\section{- To cite this version:}

Nicolas Staub, Mostafa Mohammadi, Davide Bicego, Domenico Prattichizzo, Antonio Franchi. Towards Robotic MAGMaS: Multiple Aerial-Ground Manipulator Systems. 2017 IEEE International Conference on Robotics and Automation, May 2017, Singapore, Singapore. hal-01476813

\section{HAL Id: hal-01476813 https://hal.laas.fr/hal-01476813}

Submitted on 25 Feb 2017

HAL is a multi-disciplinary open access archive for the deposit and dissemination of scientific research documents, whether they are published or not. The documents may come from teaching and research institutions in France or abroad, or from public or private research centers.
L'archive ouverte pluridisciplinaire HAL, est destinée au dépôt et à la diffusion de documents scientifiques de niveau recherche, publiés ou non, émanant des établissements d'enseignement et de recherche français ou étrangers, des laboratoires publics ou privés. 


\title{
Towards Robotic MAGMaS: Multiple Aerial-Ground Manipulator Systems
}

\author{
Nicolas Staub ${ }^{1}$, Mostafa Mohammadi ${ }^{2,3}$, Davide Bicego ${ }^{1}$, Domenico Prattichizzo ${ }^{2,3}$ and Antonio Franchi ${ }^{1}$
}

\begin{abstract}
In this paper we lay the foundation of the first heterogeneous multi-robot system of the Multiple AerialGround Manipulator System (MAGMaS) type. A MAGMaS consists of a ground manipulator and a team of aerial robots equipped with a simple gripper manipulator the same object. The idea is to benefit from the advantages of both kinds of platforms, i.e., physical strength versus large workspace. The dynamic model of such robotic systems is derived, and its characteristic structure exhibited. Based on the dynamical structure of the system a nonlinear control scheme, augmented with a disturbance observer is proposed to perform trajectory tracking tasks in presence of model inaccuracies and external disturbances. The system redundancy is exploited by solving an optimal force/torque allocation problem that takes into account the heterogeneous system constraints and maximizes the force manipulability ellipsoid. Simulation results validated the proposed control scheme for this novel heterogeneous robotic system. We finally present a prototypical mechanical design and preliminary experimental evaluation of a MAGMaS composed by a kuka LWR4 and quadrotor based aerial robot.
\end{abstract}

\section{INTRODUCTION}

Strong results on the control of small-size multi-rotor aerial robots (AR) in free flight have been demonstrated [1], [2]. So far, these vehicles have mainly been used in applications such as patrolling and visual inspection. The next step in the natural development of these systems is to consider their usage for physical interaction with the environment, known as aerial manipulation The recent related European research projects, such as AIRobots, ARCAS, AEROworks and AeRoArms, have pushed forward the state of art in aerial manipulation significantly, Aerial manipulation can be beneficial in a wide range of applications, especially in remote locations or for tasks located high from the ground.

So far the proposed solutions in aerial manipulation can be separated in two groups, considering a single AR or a fleet of them. In the first case, a unique vehicle is used to perform the task, as in, e.g., [3]. The usual limitations for those applications are the limited payload of small ARs [4] or the complexity of bigger unmanned aerial vehicles [3]. Moreover, the typical underactuation of such aerial platforms restrains the variety of possible achievable tasks. On the

${ }^{1}$ LAAS-CNRS, Université de Toulouse, CNRS, Toulouse, France, nicolas.staub@laas.fr, davide.bicego@laas.fr

antonio.franchi@laas.fr

${ }^{2}$ Department of Information Engineering and Mathematics, University of Siena, via Roma 56, 53100 Siena, Italy, mohammadiedii.unisi.it, prattichizzo@diedii.unisi.it

${ }^{3}$ Department of Advanced Robotics, Istituto Italiano di Tecnologia, via Morego 30, 16163 Genova, Italy

This work has been partially funded by the European Union's Horizon 2020 research and innovation programme under grant agreement No 644271 AEROARMS

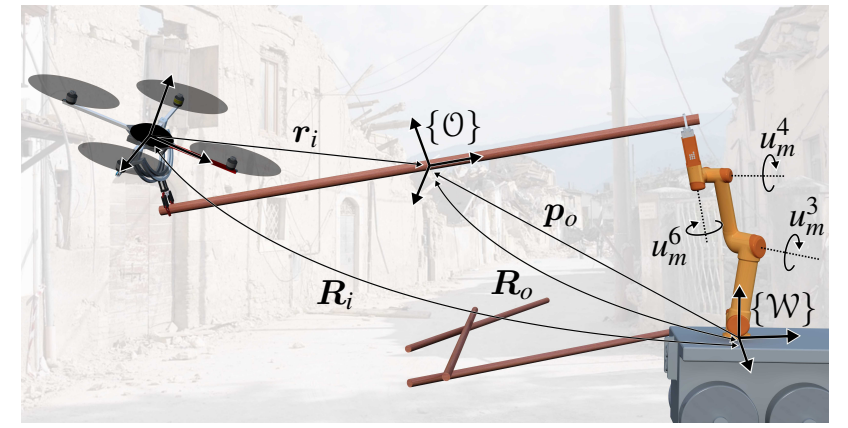

Fig. 1: Representation of a MAGMaS, a heterogeneous multi-robot system composed by a ground manipulator and one aerial robot, in a USAR scenario. Overlaid are the main coordinate frames, states, and control inputs used in the dynamic modeling.

other hand, the use of a fleet of small ARs used as a flying crane [5] has been demonstrated, the dynamics of the load is taken into account and the total wrench produced by the ARs is significantly higher. Also a group of ARs can be used to carry a load by directly attaching to the load [6] or mimicking the grasping of a hand [7], [8]. More toward the aerial manipulator paradigm, the use of a group of small ARs to carry a tool and use it to physically interact with the environment has been proposed in [9]. Those approaches consider autonomous fleet of homogeneous underactuated vehicles, resolving the under actuation limitation, but the small payload and endurance issues remain.

In this paper in order to overcome the payload issue we use a robotic (mobile) manipulators, that has a considerably higher payload compared to small ARs, and this comes with no cost on the endurance side. The assistance provided by an AR or a fleet of them complements the natural imperfection of grounded robotic manipulators in dealing with large objects, and also expands their workspace.

Consider for example a robotic manipulator tasked to let a large object track a planned trajectory. This task can become unfeasible for some kind of objects or objects/trajectories combination, e.g., with long objects requiring a lot of torque for horizontal pick-up, or precise manipulation of long objects grasped at one end, or for flexible objects. More specifically consider the task of inserting a long bar into the ceiling, to do that a manipulator will typically need assistance in two parts of the task. First for lifting the bar held by one of its extremity, as even a lightweight bar generates considerable torques when rotated at one of its end. Assistance will also be needed to precisely position the bar into a hole in the ceiling, as a small angular displacement at the grasping point will result in a large motion at the other end. In these and many other situations, such as Urban Search And Rescue 
(USAR) scenarios, see Fig. 1, an aerial manipulator, or a fleet of them can improve the precision of the tracking task, and ensure multiple contacts thus allowing to neglect the object flexibility. We use the term 'MAGMaS' (Multiple Aerial-Ground Manipulator System) to refer to these kind of multi-robot systems. To the best of our knowledge, this work presents the first complete analysis, control, and redundancy exploitation for a multi-robot team of the MAGMaS type. Our contribution is twofold: first we derive the model of a robotic system composed of a manipulator and a fleet of aerial robots. Then we propose a nonlinear control scheme, that considers existing uncertainties of the model and system constraints, and optimizes the Force Manipulability Index for trajectory tracking tasks. The proposed approach is finally validated through realistic numerical simulations and a preliminary real-world experiment.

The rest of the paper is organized as follows, Sec. II presents the system dynamic model, and Sec. III explains the controller design. Numerical simulations are then presented in Sec. IV and preliminary experimental results in Sec. V. Finally, in Sec. VI we comment our results and give a future perspective.

\section{PRoblem Formulation}

We consider a system composed by an $n$-DoF ground manipulator, and $k$ aerial robots (ARs) that cooperatively manipulate an object (see Fig. 1 where only one AR is displayed). The end-effector of the ground manipulator is equipped with a gripper in order to rigidly grasps the object. Each AR is equipped with a grasping link attached to the Center of Mass (CoM) by means of a passive spherical joint. At the other end, the grasping link is equipped with a gripper. This mechanism allows to grasp the object while leaving the AR attitude unconstrained.

Let $\mathcal{W}:\left\{O_{\mathcal{W}}, \boldsymbol{x}_{w}, \boldsymbol{y}_{w}, \boldsymbol{z}_{z}\right\}$ be the world frame, an let the object body frame be denoted with $\mathcal{O}:\left\{\boldsymbol{O}_{\mathcal{O}}, \boldsymbol{x}_{o}, \boldsymbol{y}_{o}, \boldsymbol{z}_{o}\right\}$, where $O_{\mathcal{O}}$ is the object CoM. Without loss of generality we assume that $\mathcal{O}$ is parallel to the arm end-effector frame. The body frame of the $i$-th AR (with $i \in\{1, \ldots, k\}$ ) is denoted with $\mathcal{O}_{i}:\left\{O_{i}, \boldsymbol{x}_{i}, \boldsymbol{y}_{i}, \boldsymbol{z}_{i}\right\}$ where $O_{i}$ is the AR CoM.

We denote with $\boldsymbol{p}_{o}, \boldsymbol{p}_{i} \in \mathbb{R}^{3}$, and $\boldsymbol{R}_{o}, \boldsymbol{R}_{i} \in S O(3)$ the position of $O_{\mathcal{O}}$ and $O_{i}$, and the rotation matrices expressing the orientation of $\mathcal{O}$ and $\mathcal{O}_{i}$ w.r.t. $\mathcal{W}$, respectively. The matrices $\boldsymbol{R}_{o}$ and $\boldsymbol{R}_{i}$ are parameterized by a set of roll/pitch/yaw angles $\boldsymbol{\eta}_{o}=\left[\begin{array}{lll}\phi_{o} & \theta_{o} & \psi_{o}\end{array}\right]^{\top} \in \mathbb{R}^{3}$ and $\boldsymbol{\eta}_{i}=\left[\begin{array}{lll}\phi_{i} & \theta_{i} & \psi_{i}\end{array}\right]^{\top} \in \mathbb{R}^{3}$, respectively. The angular velocities $\mathcal{O}$ and $\mathcal{O}_{i}$ w.r.t. $\mathcal{W}$, expressed in the corresponding body frame, are denoted with $\boldsymbol{\omega}_{o}, \boldsymbol{\omega}_{i} \in \mathbb{R}^{3}$, respectively. Furthermore, let $m_{o}, m_{i} \in \mathbb{R}^{+}$and $\boldsymbol{J}_{o}, \quad \boldsymbol{J}_{i} \in \mathbb{R}^{3 \times 3}$ be the mass and inertia matrix of the object and $i$-th AR. The key quantities are summarized in Tab. I.

The $i$-th aerial robot exerts a thrust force $\boldsymbol{u}_{t}^{i}=u_{t}^{i} z_{i}$ applied at its CoM with a controllable magnitude $u_{t}^{i}$ and a direction $z_{i}$ that is specified by the AR orientation, which is regulated by the control torque vector $\boldsymbol{u}_{r}^{i}=\left[\begin{array}{lll}u_{x}^{i} & u_{y}^{i} & u_{z}^{i}\end{array}\right]^{\top} \in \mathbb{R}^{3}$. The dynamic equation of motion for each AR is as follow

$$
\begin{aligned}
m \ddot{p}_{i}+m_{i} g \boldsymbol{z}_{w} & =u_{t}^{i} \boldsymbol{R}_{i} \boldsymbol{z}_{b}^{i}-\boldsymbol{h}_{i} \\
\boldsymbol{J}_{i} \dot{\omega}_{i}+\boldsymbol{\omega}_{i} \times \boldsymbol{J}_{i} \boldsymbol{\omega}_{i} & =\boldsymbol{u}_{r}^{i}
\end{aligned}
$$

where $\boldsymbol{h}_{i} \in \mathbb{R}^{3}$ is the load of the system on $i$-th AR. From (2), recalling the relationship between $\boldsymbol{\omega}_{i}$, and the derivative the Euler angles $\dot{\boldsymbol{\eta}}_{i}$ as $\boldsymbol{\omega}_{i}=\boldsymbol{E}_{i}\left(\boldsymbol{\eta}_{i}\right) \dot{\boldsymbol{\eta}}_{i}$, the rotational dynamics of the $i$-th AR is

$$
\boldsymbol{M}_{i} \ddot{\boldsymbol{\eta}}_{i}+\boldsymbol{c}_{i}\left(\boldsymbol{\eta}_{i}, \dot{\boldsymbol{\eta}}_{i}\right)=\boldsymbol{u}_{r}^{i}
$$

in which $\boldsymbol{M}_{i} \in \mathbb{R}^{3 \times 3}$ is the rotational part of the $i$-th aerial robot's Inertia matrix, $\boldsymbol{c}_{i}\left(\boldsymbol{\eta}_{i}, \dot{\boldsymbol{\eta}}_{i}\right) \in \mathbb{R}^{3}$ is the Coriolis/centripetal term. The position of $O_{i}$ in $\mathcal{O}$ is denoted by $\boldsymbol{r}_{i} \in \mathbb{R}^{3}$. Thus we have $\boldsymbol{p}_{i}=\boldsymbol{p}_{o}+\boldsymbol{R}_{o} \boldsymbol{r}_{i}$ for $i \in\{1, \ldots, k\}$.

The dynamical model of the ground manipulator is

$$
\boldsymbol{M}_{m}\left(\boldsymbol{q}_{m}\right) \ddot{\boldsymbol{q}}_{m}+\boldsymbol{c}_{m}\left(\boldsymbol{q}_{m}, \dot{\boldsymbol{q}}_{m}\right)+\boldsymbol{g}_{m}\left(\boldsymbol{q}_{m}\right)=\boldsymbol{u}_{m}-\boldsymbol{J}_{m}^{\top}\left(\boldsymbol{q}_{m}\right) \boldsymbol{h}_{o}
$$

where $\boldsymbol{q}_{m}=\left[\begin{array}{lll}q_{1} & \ldots & q_{n}\end{array}\right]^{\top} \in \mathbb{R}^{n}$ is the joint angle coordinate vector, $\boldsymbol{h}_{o} \in \mathbb{R}^{6}$ is the wrench applied by the arm to the object and ARs system, expressed in the end-effector frame, $\boldsymbol{M}_{m}\left(\boldsymbol{q}_{m}\right) \in \mathbb{R}^{n \times n}$ is the inertia matrix, $\boldsymbol{c}_{m}\left(\boldsymbol{q}_{m}, \dot{\boldsymbol{q}}_{m}\right) \in \mathbb{R}^{n}$ and $\boldsymbol{g}_{m}\left(\boldsymbol{q}_{m}\right) \in \mathbb{R}^{n}$ represent the Coriolis/centripetal and gravity terms, respectively, $\boldsymbol{J}_{m}\left(\boldsymbol{q}_{m}\right) \in \mathbb{R}^{6 \times n}$ is the geometric Jacobian of the arm, and $\boldsymbol{u}_{m}=\left[u_{m}^{1} \ldots u_{m}^{n}\right]^{\top} \in \mathbb{R}^{n}$ gathers the $n$ joint torques of the manipulator.

Considering $\boldsymbol{q}=\left[\begin{array}{ll}\boldsymbol{q}_{m}^{\top} & \boldsymbol{q}_{a}^{\top}\end{array}\right]^{\top} \in \mathbb{R}^{n+3 k}$, where $\boldsymbol{q}_{a}=$ $\left[\begin{array}{llll}\boldsymbol{\eta}_{1}^{\top} & \ldots & \boldsymbol{\eta}_{k}^{\top}\end{array}\right]^{\top} \in \mathbb{R}^{3 k}$, and $\boldsymbol{u}=\left[\begin{array}{ll}\boldsymbol{u}_{m}^{\top} & \boldsymbol{u}_{r}^{\top}\end{array}\right]^{\top} \in \mathbb{R}^{n+3 k}$ in which $\boldsymbol{u}_{r}=\left[\boldsymbol{u}_{r}^{1^{\dagger}} \ldots \boldsymbol{u}_{r}^{k^{\top}}\right]^{\top} \in \mathbb{R}^{3 k}$, the dynamical model of the whole system can be written as

$$
\boldsymbol{M}(\boldsymbol{q}) \ddot{\boldsymbol{q}}+\boldsymbol{c}(\boldsymbol{q}, \dot{\boldsymbol{q}})+\boldsymbol{g}(\boldsymbol{q})=\boldsymbol{u}-\boldsymbol{J}^{\top}(\boldsymbol{q}) \boldsymbol{h},
$$

in which $\boldsymbol{M}(\boldsymbol{q})=\operatorname{diag}\left(\boldsymbol{M}_{m}\left(\boldsymbol{q}_{m}\right), \boldsymbol{M}_{1}\left(\boldsymbol{\eta}_{1}\right), \ldots, \boldsymbol{M}_{k}\left(\boldsymbol{\eta}_{k}\right)\right)$, $\boldsymbol{c}(\boldsymbol{q}, \dot{\boldsymbol{q}})=\left[\boldsymbol{c}_{m}\left(\boldsymbol{q}_{m}, \dot{\boldsymbol{q}}_{m}\right) \boldsymbol{c}_{1}\left(\boldsymbol{\eta}_{1}, \dot{\boldsymbol{\eta}}_{1}\right) \cdots \boldsymbol{c}_{k}\left(\boldsymbol{\eta}_{k}, \dot{\boldsymbol{\eta}}_{k}\right)\right]^{\top}$ and $\boldsymbol{g}(\boldsymbol{q})=$ $\left[\boldsymbol{g}_{m}\left(\boldsymbol{q}_{m}\right) \mathbf{0}_{3 k \times 1}\right]^{\top}$, and $\boldsymbol{J}=\operatorname{diag}\left\{\boldsymbol{J}_{m}\left(\underline{q}_{m}\right), \mathbf{0}_{3}, \ldots, \mathbf{0}_{3}\right\}$. The term $\boldsymbol{h} \in \mathbb{R}^{6+3 k}$ is defined as $\boldsymbol{h}=\left[\begin{array}{ll}\boldsymbol{h}_{o}^{\top} & \boldsymbol{h}_{t}^{\top}\end{array}\right]^{\top}$, where $\boldsymbol{h}_{t}=$ $\left[\boldsymbol{h}_{1}^{\top} \ldots, \boldsymbol{h}_{k}^{\top}\right]^{\top}$. The structure of $\boldsymbol{J}$ arises from the fact that the passive joints efficiently decouple the ARs rotational dynamics. The dynamic equation of motion for the rigid body object completes the dynamic model of the system

$$
\boldsymbol{M}_{o}(\boldsymbol{x}) \ddot{\boldsymbol{x}}+\boldsymbol{c}_{o}(\boldsymbol{x}, \dot{\boldsymbol{x}})+\boldsymbol{g}_{o}(\boldsymbol{x})=\boldsymbol{h}_{e}
$$

where $\boldsymbol{x}=\left[\begin{array}{ll}\boldsymbol{p}_{o}^{\top} & \boldsymbol{\eta}_{o}^{\top}\end{array}\right]^{\top} \in \mathbb{R}^{6}$ is the object pose vector, $\boldsymbol{M}_{o} \in \mathbb{R}^{6}, \boldsymbol{c}_{o} \in \mathbb{R}^{6}$, and $\boldsymbol{g}_{o} \in \mathbb{R}^{6}$ are inertial matrix, Coriolis/centripetal, and gravity vectors, respectively, and $\boldsymbol{h}_{e}$, called external wrench, is the resultant force of the arm manipulator and all the ARs that moves the object, and can be calculated as follows

$$
\boldsymbol{h}_{e}=\boldsymbol{G} \boldsymbol{h}
$$

where the grasp matrix $\boldsymbol{G}$ is defined as $\boldsymbol{G}=\left[\begin{array}{ll}\boldsymbol{T}_{m} & \boldsymbol{G}_{t}(\boldsymbol{q})\end{array}\right]$ in which $\boldsymbol{T}_{m} \in \mathbb{R}^{6 \times 6}$ transforms $\boldsymbol{h}_{o}$ from the end-effector frame to the world frame, and $\boldsymbol{G}_{t}(\boldsymbol{q}) \in \mathbb{R}^{6 \times 3 k}$ describes the influence of the aerial robot thrust vectors on the object motion. It is straightforward to obtain them as follows

$$
\begin{aligned}
\boldsymbol{T}_{m} & =\left[\begin{array}{cc}
\boldsymbol{R}_{o}^{\top} & \mathbf{0} \\
\boldsymbol{S}\left(\boldsymbol{R}_{o}^{\top} \boldsymbol{r}_{e}\right) & \boldsymbol{R}_{o}^{\top}
\end{array}\right] \\
\boldsymbol{G}_{t}(\boldsymbol{q}) & =\left[\begin{array}{ccc}
\boldsymbol{I}_{3} & \ldots & \boldsymbol{I}_{3} \\
\boldsymbol{S}\left(\boldsymbol{R}_{o}^{\top} \boldsymbol{r}_{i}\right) & \ldots & \boldsymbol{S}\left(\boldsymbol{R}_{o}^{\top} \boldsymbol{r}_{k}\right)
\end{array}\right]
\end{aligned}
$$

where $\boldsymbol{r}_{e} \in \mathbb{R}^{3}$ is the end effector position in $\mathcal{O}$ and $\boldsymbol{S}$ the the skew-symmetric operator on a vector. Now consider (7), 


\begin{tabular}{|c|c|}
\hline notations & description \\
\hline 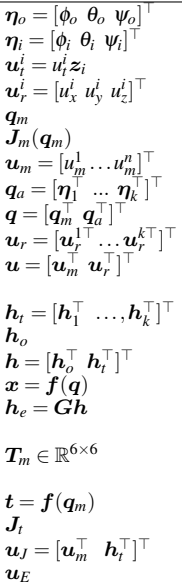 & $\begin{array}{l}\text { roll/pitch/yaw (RPY) angles of the object } \mathcal{O} \\
\text { RPY angles of the } i \text {-th Aerial Robot (AR) } \\
\text { thrust vector of the } i \text {-th AR } \\
\text { input torque of the } i \text {-th AR } \\
\text { joint positions of the ground manipulator } \\
\text { geometric Jacobian of the ground manipulator } \\
\text { ground manipulator input torques } \\
\text { all ARs orientations } \\
\text { coordinates of the system } \\
\text { torque of all ARs } \\
\text { input torques of the ARs and the ground } \\
\text { manipulator } \\
\text { load of the system on each AR } \\
\text { wrench applied by the arm to }\{\text { object }+ \text { ARs\} } \\
\text { coupling wrenches in the system } \\
\text { Cartesian coordinate of the object } \mathcal{O} \\
\text { resultant wrench of the arm and all the ARs } \\
\text { that moves the object } \mathcal{O} \\
\text { mapping for wrench applied to }\{\text { object }+ \\
\text { ARs\} from end-effector frame to } \mathcal{W} \text {-frame } \\
\text { task function } \\
\text { Jacobian matrix of the task } \\
\text { ground manipulator torque and ARs forces } \\
\text { feedback-linearization-based control signal }\end{array}$ \\
\hline
\end{tabular}

TABLE I: Main notations used through the paper.

the grasp matrix $\boldsymbol{G}$ is full-row rank, thus for a given $\boldsymbol{h}_{e}$ the inverse of (7) can be written as follows

$$
\boldsymbol{h}=\boldsymbol{G}^{+} \boldsymbol{h}_{e}+\boldsymbol{V} \boldsymbol{h}_{n}=\boldsymbol{h}_{E}+\boldsymbol{h}_{\boldsymbol{I}}
$$

where $\boldsymbol{G}^{+}$is a pseudo-inverse of $\boldsymbol{G}$, and $\boldsymbol{V}$ is a full-row rank matrix spanning the null-space of $\boldsymbol{G}$, and $\boldsymbol{h}_{n}$ is an arbitrary vector of appropriate dimension that parameterizes the solution sets [10]. Concatenated wrench vector $\boldsymbol{h}_{E}$ are wrenches that can result in motion, while $\boldsymbol{h}_{I}$ are known as internal wrenches, and their directions are such that they do not contribute to a motion.

\section{A. System Constraints}

The ground manipulator joint torques and force vector of each AR must comply with the following system constraints.

- The limited rotations of spherical joints constrains the force applied by the $i$-th AR to the object.

$$
\chi_{i}\left(\boldsymbol{\eta}_{i}\right)=\sqrt{\left(h_{i}^{x}\right)^{2}+\left(h_{i}^{y}\right)^{2}}-\tan \left(\alpha_{i}\right) h_{i}^{z} \leq 0
$$

where $\boldsymbol{h}_{i}=\left[h_{i}^{x}, h_{i}^{y}, h_{i}^{z}\right]^{\top}$ is the force vector, and $\alpha_{i} \in \mathbb{R}^{+}$ shows the allowed cone angle of a spherical joint;

- the ground manipulator joints have limited rotation range,

$$
q_{i}^{\min } \leq q_{i} \leq q_{i}^{\max } \quad i=1, \ldots, n
$$

where $q_{i}^{\min }, q_{i}^{\max } \in \mathbb{R}^{+}$are scalar values representing the upper and lower joint bounds;

- the robot manipulator torques are limited,

$$
u_{m}^{i^{\min }} \leq u_{m}^{i} \leq u_{m}^{i_{\max }} \quad i=1, \ldots, n
$$

where $u_{m}^{i^{\min }}, u_{m}^{i^{\max }} \in \mathbb{R}$ represent the upper and lower torque bounds for the manipulator;

- each AR has a bounded thrust,

$$
\left\|\boldsymbol{h}_{i}\right\| \leq h_{i}^{\max } \quad i=1, \ldots, k
$$

where $h_{i}^{\max } \in \mathbb{R}^{+}$is the maximum applicable thrust.

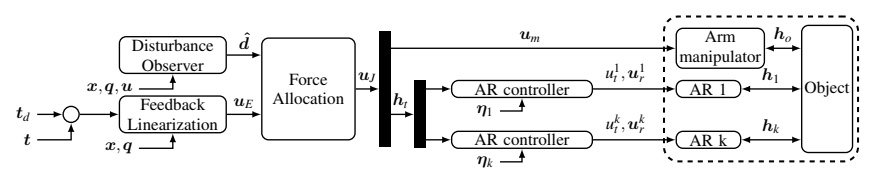

Fig. 2: Proposed control scheme for MAGMaS (dashed rectangle), composed of a feedback linearization controller, a disturbance observer, an optimization-based force allocation scheme and one AR force vector controllers for each aerial manipulator.

\section{CONTROL}

This section details the control architecture, derives the feedback linearization, formalizes the force allocation optimization problem and uncertainties handling is introduced. The overall control architecture is summarized in Fig. 2.

Consider a task associated to the manipulation of the object, such as trajectory tracking in operational workspace. The task is described by a set of variables $t \in \mathbb{R}^{\sigma}$, since the object is a rigid body $\sigma \leq 6$. On the other hand, the object configuration only depends on the arm joint angles $\boldsymbol{q}_{m}$, thus the task is function of the sole $\boldsymbol{q}_{m}$, and can be written $\boldsymbol{t}=\boldsymbol{f}\left(\boldsymbol{q}_{m}\right)$, where $\boldsymbol{f}: \mathbb{R}^{n} \rightarrow \mathbb{R}^{\sigma}$ is a differentiable map assumed to be known for a given manipulator. A prerequisite for a generic reference to be trackable is that the map $f$ is surjective, which, in turns, implies $\sigma \leq n$. In conclusion, $\sigma \leq \min \{6, n\}$. Furthermore, we assume that the task is planned such as to comply with the robot manipulator joint limits (12). The problem addressed in this work is to let the task $\boldsymbol{t}$ track a desired reference $\boldsymbol{t}_{\boldsymbol{d}}$ while taking advantage of the MAGMaS redundancy and heterogeneity.

The trajectory tracking task control is done through inputoutput exact linearization, via static feedback. Recall, in order to design a static feedback linearization control law each output is differentiated until at least one input appears and the obtained differential map must result invertible. In our case, the first differentiation of the task w.r.t. time yields

$$
\dot{\boldsymbol{t}}=\frac{\partial \boldsymbol{f}}{\partial \boldsymbol{q}_{m}} \dot{\boldsymbol{q}}_{m}=\boldsymbol{J}_{t}\left(\boldsymbol{q}_{m}\right) \dot{\boldsymbol{q}}_{m}=\left[\boldsymbol{J}_{t}\left(\boldsymbol{q}_{\boldsymbol{m}}\right) \mathbf{0}_{\sigma_{t} \times 3 k}\right] \dot{\boldsymbol{q}}
$$

where $J_{t} \in \mathbb{R}^{\sigma \times n}$ is known as task Jacobian. A second time differentiation is necessary to make the control inputs appear,

$$
\ddot{\boldsymbol{t}}=\frac{\partial \dot{\boldsymbol{f}}}{\partial \boldsymbol{q}_{m}} \dot{\boldsymbol{q}}_{m}+\boldsymbol{J}_{t}\left(\boldsymbol{q}_{m}\right) \ddot{\boldsymbol{q}}_{m}=\boldsymbol{f}_{t}\left(\boldsymbol{q}_{m}, \dot{\boldsymbol{q}}_{m}, \ddot{\boldsymbol{q}}_{m}\right)+\boldsymbol{G}_{u}(\boldsymbol{q}) \boldsymbol{u}_{J}
$$

where

$$
\begin{array}{r}
\boldsymbol{f}_{t}\left(\boldsymbol{q}_{m}, \dot{\boldsymbol{q}}_{m}, \ddot{\boldsymbol{q}}_{m}\right)=\frac{\partial \dot{\boldsymbol{f}}}{\partial \boldsymbol{q}_{m}} \dot{\boldsymbol{q}}_{m}-\boldsymbol{J}_{t} \boldsymbol{M}_{m}^{-1}\left(\boldsymbol{c}_{m}\left(\boldsymbol{q}_{m}, \dot{\boldsymbol{q}}_{m}\right)+\boldsymbol{g}_{m}\left(\boldsymbol{q}_{m}\right)\right)- \\
\boldsymbol{J}_{t} \boldsymbol{M}_{m}^{-1} \boldsymbol{J}_{m}^{\top} \boldsymbol{T}_{m}^{-1}\left(\boldsymbol{M}_{o}(\boldsymbol{x}) \ddot{\boldsymbol{x}}+\boldsymbol{c}_{o}(\boldsymbol{x}, \dot{\boldsymbol{x}})+\boldsymbol{g}_{o}(\boldsymbol{x})\right) \\
\boldsymbol{G}_{u}(\boldsymbol{q})=\boldsymbol{J}_{t}\left(\boldsymbol{q}_{m}\right) \boldsymbol{M}_{m}^{-1}\left(\boldsymbol{q}_{m}\right) \underbrace{\left[\begin{array}{ll}
\boldsymbol{I}_{n} & \left.\boldsymbol{J}_{m}^{\top} \boldsymbol{T}_{m}^{-1} \boldsymbol{G}_{t}(\boldsymbol{q})\right]
\end{array}\right]}_{\boldsymbol{A}}
\end{array}
$$

and $\boldsymbol{u}_{J}=\left[\begin{array}{ll}\boldsymbol{u}_{m}^{\top} & \boldsymbol{h}_{t}^{\top}\end{array}\right]^{\top}$. Given our assumptions, the matrix $\boldsymbol{G}_{u}$ is full row-rank whenever $\boldsymbol{J}_{t}$ is full row-rank, because by construction $\boldsymbol{A}$ is the projection of $\boldsymbol{u}_{J}$ on the manipulator joints and $M_{m}^{-1}$ is full rank by definition. From the structure it is also clear that the inputs directly related with the task dynamics are the manipulator torques, $\boldsymbol{u}_{m}$, and the concatenated force vector $\boldsymbol{h}_{t}$, generated by the ARs, through 
(1)-(2). Task trajectory tracking can be enforced by a control action $\boldsymbol{u}_{E} \in \mathbb{R}^{\sigma}$ such that

$$
\boldsymbol{u}_{E}=\ddot{\boldsymbol{t}}_{d}+\boldsymbol{K}_{D} \dot{\boldsymbol{e}}+\boldsymbol{K}_{P} \boldsymbol{e}-\boldsymbol{f}_{t}
$$

where $\boldsymbol{e}=\boldsymbol{t}_{d}-\boldsymbol{t}, \boldsymbol{K}_{D} \in \mathbb{R}^{\sigma \times \sigma}$ and $\boldsymbol{K}_{P} \in \mathbb{R}^{\sigma \times \sigma}$ are diagonal positive definite matrices. To implement $\boldsymbol{u}_{E}, \boldsymbol{u}_{J}$ has to verify

$$
\hat{\boldsymbol{G}}_{u} \boldsymbol{u}_{J}=\boldsymbol{u}_{E},
$$

in order to be plugged in (16) to ensure the tracking of a desired trajectory $\boldsymbol{t}_{d}$. Thanks to redundancy there are infinite possible input allocations, $\boldsymbol{u}_{J}$, for a given $\boldsymbol{u}_{E}$.

To do so, we formulate the control problem as a programming problem to minimize the cost function $\mathcal{J}: \mathbb{R}^{(n+3 k)} \mapsto \mathbb{R}$ defined as $\mathcal{J}\left(\boldsymbol{u}_{J}\right)=\boldsymbol{u}_{J}^{\top} \boldsymbol{P} \boldsymbol{u}_{J}$, where $\boldsymbol{P} \in \mathbb{R}^{(n+3 k) \times(n+3 k)}$, defined as $\boldsymbol{P}=\operatorname{diag}\left\{\boldsymbol{J}_{t} \boldsymbol{J}_{t}^{\top}, \boldsymbol{P}_{t}\right\}$, is a weighting matrix to allocate the forces according to the maximum torque of the ground manipulator motors and AR thrusters, and in order to increase the force manipulability ellipsoid described by the matrix $\boldsymbol{J}_{t} \boldsymbol{J}_{t}^{T}$. The matrix $\boldsymbol{P}_{t} \in \mathbb{R}^{3 k \times 3 k}$ allows to weight the ARs differently from each other and from the ground manipulator. The solution of the optimization problem is constrained by $\boldsymbol{u}_{J} \in \mathcal{F}$, where $\mathcal{F}$ is the feasible solutions set defined by the inequalities (11), (13), and (14), plus $\boldsymbol{u}_{J}$ should satisfy (18) which yields the constraint, $\boldsymbol{\xi}\left(\boldsymbol{u}_{J}\right)=$ $\boldsymbol{G}_{u} \boldsymbol{u}_{J}-\boldsymbol{u}_{E}=0$, where $\boldsymbol{\xi}: \mathbb{R}^{n+3 k} \mapsto \mathbb{R}^{\sigma}$.

Note that the constraint (12) is addressed by the task choice. In summary, the control allocation problem is

$$
\begin{array}{rl}
\boldsymbol{u}_{J}^{*}=\arg \min _{\boldsymbol{u}_{J}} & \mathcal{J}\left(\boldsymbol{u}_{J}\right) \\
\text { s.t. } & \chi_{i}\left(\boldsymbol{\eta}_{i}\right) \leq 0 \quad i=1, \ldots, k \\
& \left\|\boldsymbol{h}_{i}\right\| \leq h_{i}^{\max } \quad i=1, \ldots, k \\
& \min \left(u_{m}^{i}\right) \leq u_{m}^{i} \leq \max \left(u_{m}^{i}\right) \quad i=1, \ldots, n \\
& \boldsymbol{\xi}\left(\boldsymbol{u}_{\boldsymbol{J}}\right)=0 .
\end{array}
$$

All bounded constraints, equality and non-equality, are affine functions of the optimization variable $\boldsymbol{u}_{J}$, and since $\mathcal{J}\left(\boldsymbol{u}_{J}\right)$ is convex quadratic, (19) is a convex programming problem. A wide range of efficient methods can be used to solve the problem, as described in literature of convex optimization.

Uncertainties could arise for several reasons, parametric uncertainties such as imprecise weight and length measurements, unmodeled dynamics, such as motor dynamics or the existence of unmodeled external disturbances.

All those uncertainties could also be coped with by the system redundancy by reformulating the optimization problem (19) and the trajectory tracking control $\boldsymbol{u}_{E}$ in the case of disturbances. Let and $\hat{G}_{u}$ and $\hat{f}_{t}$ be the nominal values of $G_{u}$ and $f_{t}$ that represent the existence of a lumped bounded uncertainty in the model. A disturbance term, $\boldsymbol{d}=\hat{\boldsymbol{G}}_{u}^{+}\left(\ddot{\boldsymbol{t}}-\hat{\boldsymbol{f}}_{t}\right)-\boldsymbol{u}_{J}$, can be introduced and the second order task dynamics (16) can be rewritten as

$$
\ddot{\boldsymbol{t}}=\hat{\boldsymbol{f}}_{t}+\hat{\boldsymbol{G}}_{u}\left(\boldsymbol{u}_{J}-\boldsymbol{d}\right) \text {. }
$$

A common approach to estimate the disturbance is to use the following disturbance observer

$$
\dot{\hat{d}}=-\boldsymbol{L} \hat{\boldsymbol{d}}+\boldsymbol{L}\left(\hat{\boldsymbol{G}}_{u}^{+}\left(\ddot{\boldsymbol{t}}-\hat{\boldsymbol{f}}_{t}\right)-\boldsymbol{u}_{J}\right)
$$

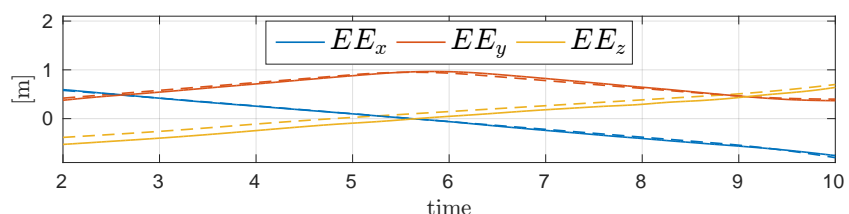

Fig. 3: Simulation case study, a ground manipulator and a AR cooperatively manipulating an object. Associated desired and actual position of the end-effector with respect to the arm base are plotted.

where $\boldsymbol{L} \in \mathbb{R}^{(n+3 k) \times(n+3 k)}$ is positive diagonal observer gain, and $\hat{d}$ is the output of the disturbance observer. This means that (18) rewrites as $\boldsymbol{u}_{E}=\hat{\boldsymbol{G}}_{u}\left(\boldsymbol{u}_{J}-\hat{\boldsymbol{d}}\right)$, and hence the optimization problem (19) as to be updated with

$$
\boldsymbol{\xi}\left(\boldsymbol{u}_{J}\right)=\hat{\boldsymbol{G}}_{u}\left(\boldsymbol{u}_{J}-\hat{\boldsymbol{d}}\right)-\boldsymbol{u}_{E}
$$

to take into account the disturbances. Using this control scheme we ensure task trajectory tracking, while the system redundancies are exploited to satisfy the system constraints and reject possible disturbances.

\section{A. AR Thurst Control}

As per the thrust controller, we utilize the following

$$
\begin{aligned}
u_{t}^{i} & =\boldsymbol{z}_{i}^{\top} \boldsymbol{R}_{i}^{\top}\left(m_{i} g \boldsymbol{z}_{w}+\boldsymbol{h}_{i}\right) \\
\boldsymbol{u}_{r}^{i} & =-\boldsymbol{K}_{R} \boldsymbol{e}_{R}^{i}-\boldsymbol{K}_{\boldsymbol{\omega}} \boldsymbol{e}_{\boldsymbol{\omega}}^{i}+\boldsymbol{\omega}_{i} \times \boldsymbol{J}_{i} \boldsymbol{\omega}_{i}
\end{aligned}
$$

where $\boldsymbol{K}_{R}, \boldsymbol{K}_{\boldsymbol{\omega}} \in \mathbb{R}^{3 \times 3}$ are diagonal gain matrices with positive elements, $\boldsymbol{e}_{R}^{i}$ is orientation matrix error as

$$
\boldsymbol{e}_{R}^{i}=\frac{1}{2} \boldsymbol{S}^{-}\left(\boldsymbol{R}_{d}^{i^{\top}} \boldsymbol{R}_{i}-\boldsymbol{R}_{i}^{\top} \boldsymbol{R}_{d}^{i}\right)
$$

where $\boldsymbol{S}^{-}(\bullet)$ is the inverse operation of $\boldsymbol{S}(\bullet)$, and $\boldsymbol{e}_{\omega}^{i}$ is the angular velocity error defined as

$$
\boldsymbol{e}_{\omega}^{i}=\boldsymbol{\omega}_{i}-\boldsymbol{R}_{i}^{\top} \boldsymbol{R}_{d}^{i} \boldsymbol{\omega}_{d}^{i}
$$

where $\omega_{d}^{i} \in \mathbb{R}^{3}$ is the desired angular velocity. The desired rotation matrix $\boldsymbol{R}_{d}^{i}$ is simply obtained by calculating any rotation matrix that transforms $\boldsymbol{z}_{w}$ to $\boldsymbol{h}_{i} /\left\|\boldsymbol{h}_{i}\right\|$, i.e., aligning the thrust direction to the load of the system on the corresponding AR.

\section{NumeriCAl Simulations}

Numerical simulation for a 6 DoF ground manipulator cooperating with one quadrotor UAV is presented in this section to show the feasibility and effectiveness of the proposed scheme. The simulation has been performed in Matlab/Simulink environment with the SimMechanics modeling toolbox. The optimization problem is solved via Sequential Quadratic Programming (SQP) method. The simulation sample time is $1 \mathrm{~ms}$, and the control loop one is $10 \mathrm{~ms}$.

The simulated ground manipulator is a Universal Robot UR5, with arms length respectively, $1.0 \mathrm{~m}$ and $0.7 \mathrm{~m}$, total mass of the arm $18.4 \mathrm{~kg}$, maximum payload $5 \mathrm{~kg}$ and maximum joint torques $[150 ; 150 ; 150 ; 28 ; 28 ; 28] \mathrm{Nm}$, from base to end-effector. The simulated AR is a quadrotor of $0.50 \mathrm{~m}$ circumference actuated by four motor-propeller sets, each one can generate up to $10 \mathrm{~N}$, and the length of the arm holding the gripper is $40 \mathrm{~cm}$. We consider the spherical joints limit to be described by a cone of $\pi / 4$ half cone angle. All 


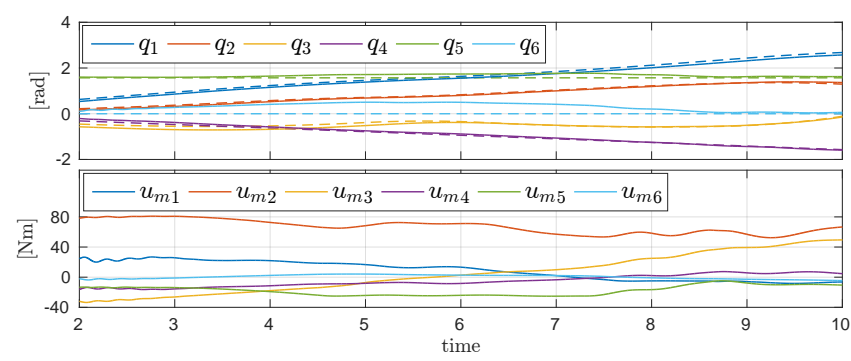

Fig. 4: Ground manipulator side. On top, both desired and actual joint angles. The desired angles are from inverse kinematics of the task trajectory. On bottom, associated manipulator input torques.

the motors are modeled as a second order linear system with a $10 \mathrm{~ms}$ rise time.

The cooperative trajectory tracking task is a trajectory tracking task with the load being a $5 \mathrm{~kg}$ bar of dimension $0.05 \mathrm{~m} \times 0.2 \mathrm{~m} \times 1 \mathrm{~m}$. We consider the loading of the bar on the back of a mobile platform on which the ground manipulator is mounted. The UR5 grasps the bar from one end and the quadrotor from the other end. The task consists to follow an appropriate trajectory (generated through waypoints and cubic-spline-based trajectory generator) to put the bar on the mobile base back. Such a scenario could be of interest in robotic search and rescue missions.

The control system is implemented considering a highly uncertain model, some terms of the controller inverse dynamic are neglected, the Inertial matrices are assumed diagonal and the Coriolis/centripetal terms are omitted. Furthermore $10 \%$ uncertainty is considered for the contact points.

The results of the trajectory tracking task are depicted in Fig. 3, with the end-effector position measured with respect to the base of the ground manipulator. As it is evident from those figures the given trajectory is tracked sufficiently well, even though the dynamics of the system is partially unknown. Note that the arm manipulator alone is not able to perform this task, because of the torque constraints. Indeed the object weight is at the limit of the ground manipulator and the weight-generated torque at the end-effector does not satisfy the joints limits because the manipulator has to grasp the object far from the its CoM.

The ground manipulator desired and actual joint angles are plotted in Fig. 4, with the desired joint angles obtained through inverse kinematics for the task trajectory. The weakness of the wrist joints generates larger errors in $\boldsymbol{q}(4,5,6)$. However, thanks to the AR support the tracking task is performed sufficiently well. The control torques of the ground manipulator, shown in Fig. 4, are far from their limits.

Fig. 5 shows the quadrotor states and control inputs in the simulation. Fig. 5 top left shows the orientation of the quadrotor which remains far away from the spherical joint limit, and Fig. 5 bottom left shows the associated control torques. Fig. 5 top right illustrates the output of the optimal force allocation algorithm for the quadrotor, that is a desired force vector $\boldsymbol{h}_{\boldsymbol{t}}$. This force vector is then generated by $\boldsymbol{u}_{\boldsymbol{r}}$ and $\boldsymbol{u}_{\boldsymbol{t}}$ which are the moments and thrust of the AR, which are shown in Fig. 5 bottom right and left, respectively, and again they all satisfy the system constraints.
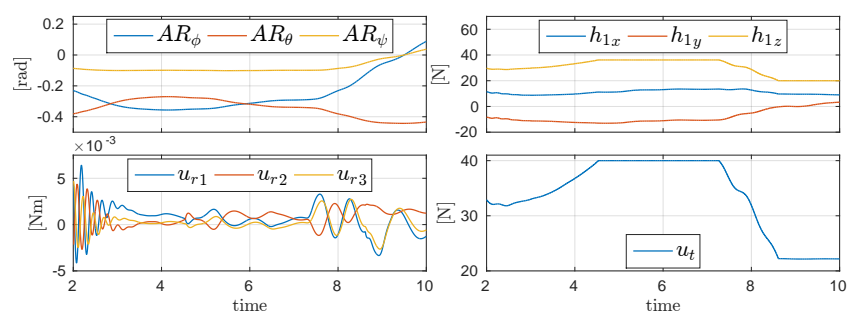

Fig. 5: AR side. Left, AR orientation and associated control torques, $\boldsymbol{u}_{\boldsymbol{r}}$. Right, output of AR force allocation $\boldsymbol{h}_{\boldsymbol{t}}$ and the AR thrust magnitude generated along AR's $\boldsymbol{z}$-axis.
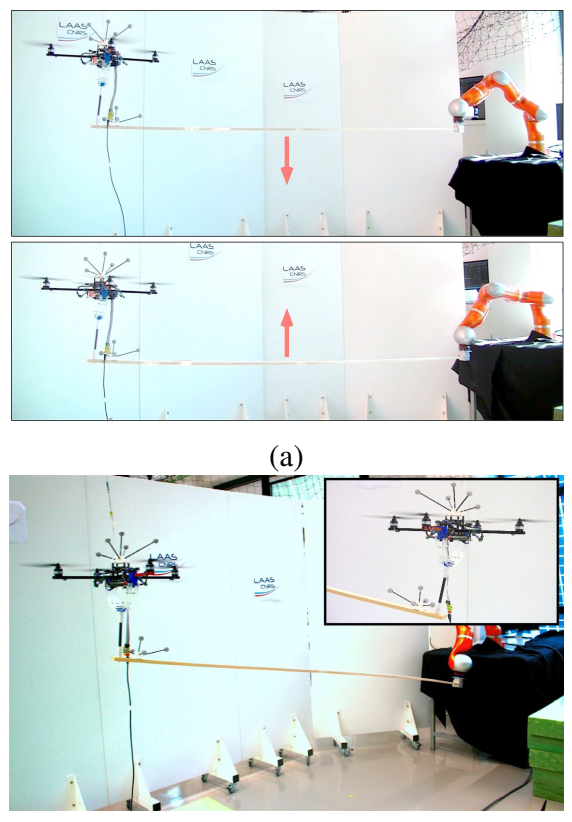

(b)

Fig. 6: Experimental set up; a kuka LWR4 arm, a classical quadrotor and an in-house designed passive rotational joint. The two extreme configurations of the up-down trajectory are depicted in $6 \mathrm{a}$ with the bar hold horizontally. Second experiment $6 \mathrm{~b}$ with the bar tilted, where the AR hovers while the kuka arm tilts the bar in order to exhibit the decoupling induced by the passive rotational joint.

\section{Preliminary EXPERIMENT}

The preliminary experimental work associated to this paper demonstrates the feasibility of MAGMaS and validates our passive rotational joint design. We consider a LWR4 kuka arm as ground manipulator and a single quadrotor as AR. The quadrotor is in-house-developed with a $1.2 \mathrm{~kg}$ payload, fitted with a custom passive rotational joint. This passive rotational joint ensures that the center of mass of the AR+joint system and the rotation center of the joint are coinciding, modulo manufacturing imperfections. This is of paramount importance as the AR can not sustain high torque disturbances. From the design, the rotational joint has the following angular constraints, two rotations are limited to $\pm 40^{\circ}$ and $\pm 80^{\circ}$ respectively and the last one is free, note that contrary to the simulation part the base of the joint cone is not a circle, but an ellipse, as the two rotations constraints are not symmetric. We choose the load to be a wooden bar of length $2.5 \mathrm{~m}$ and mass $0.61 \mathrm{~kg}$, as the focus is on the joint design validation. Also, as the grasping of the object is omitted, all sub-systems are rigidly attached 


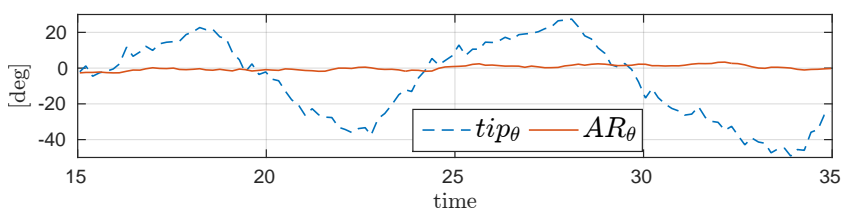

Fig. 9: orientation of the AR when subject to bar orientation changes, the passive rotational joint efficiently decouples the rotation of the AR and its end-effector attached to the bar tip.

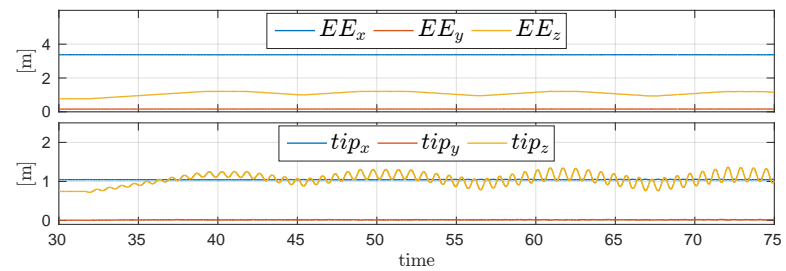

Fig. 7: The arm end-effector follows a vertical trajectory, as in Fig. 6a, without the help of an AR. The tip position vibrates a lot due to the flexibility of the bar and is not able to track the end-effector $\boldsymbol{z}$-trajectrory.

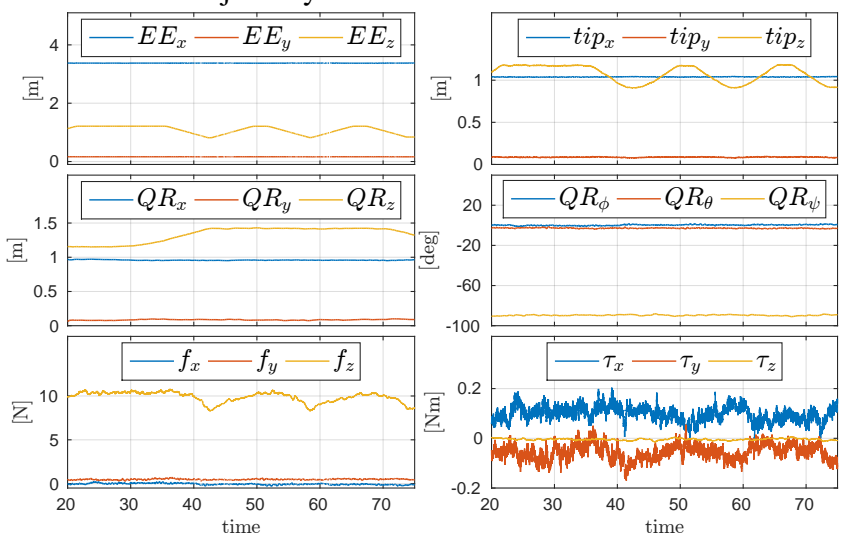

Fig. 8: The arm end-effector follows a vertical trajectory, as in Fig. 6a, in cooperation with a AR. The tip of the bar follows the $z$-trajectory of the end-effector thanks to the AR stabilizing action.

together. The full system is depicted in Fig. 6 and actual operations are featured in the attached video. As a first validation of the MAGMaS, we propose to compare the handling of the considered bar with and without the help of an AR. The end-effector is moved up and down along the $z$-axis, see fig. $6 a$ and Figs. 7-8 where the relevant quantities are plotted. Note that this comparison is possible because the bar characteristics are not violating the LWR4 payload/torques limits. Clearly the addition of the AR allows the bar tip to better follow the arm trajectory, the residual difference comes from the simple way we used to deal with the bar flexibility, proper handling of the flexibility is not addressed in this paper and would improve the performance.

A second experiment aims at validating our design of the passive rotational joint, the $\mathrm{AR}$ is commanded to remain hovering, the bar tip is then moved in order to exhibit the rational decoupling between the bar tip and the $\mathrm{QR}$, see Fig. 6b and Fig. 9 for orientation's monitoring of both tip of the bar and AR. In this experiment the orientation of the bar varies in a large range whereas the pitch of the AR remains in $\pm 3.5^{\circ}$ range, hence validating the efficiency of our passive rotational joint. Both experiments can be found in the attached video and that demonstrate the validity of our design and open path to further experimental validation.

\section{CONCLUSION}

In this paper we introduced a new kind of multi-robot system, the Multiple Aerial-Ground Manipulator System, shortly called MAGMaS. The motivation is to benefit from the advantages of both kinds of platforms, i.e., physical strength for the manipulator versus large workspace for the aerial robots, to accomplish manipulation tasks. For this new kind of system we derived the associated model, and considered a set of tasks taking advantage of the system structure. We derived a static feedback linearization control schemes which exploits the system redundancies to concurrently ensure trajectory tracking, system constraints respect and disturbances rejection. Trajectory tracking as been proven successful in realistic numerical simulations. Finally we presented experiments of a preliminary system, validating the MAGMaS approach and the mechanical design. We plan to follow on this work by considering an increasing number of aerial robots. We want to study their influence on the cost function, but also on the offset of the ellipse center and how this could be optimized for the tasks considered. We also plan to conduct full experiments with a real system composed of several ARs.

\section{REFERENCES}

[1] R. Mahony, V. Kumar, and P. Corke, "Multirotor Aerial Vehicles: Modeling, Estimation, and Control of Quadrotor," IEEE Robotics \& Automation Magazine, vol. 19, no. 3, pp. 20-32, 2012.

[2] A. Franchi, C. Secchi, M. Ryll, H. H. Bülthoff, and P. Robuffo Giordano, "Shared control: Balancing autonomy and human assistance with a group of quadrotor UAVs," IEEE Robotics \& Automation Magazine, Special Issue on Aerial Robotics and the Quadrotor Platform, vol. 19, no. 3, pp. 57-68, 2012.

[3] K. Kondak, F. Hubert, M. Schwarzbach, M. Laiacker, D. Sommer, M. Bejar, and A. Ollero, "Aerial manipulation robot composed of an autonomous helicopter and a 7 degrees of freedom industrial manipulator," in 2014 IEEE Int. Conf. on Robotics and Automation, Hong Kong, China, May 2014, pp. 2108-2112.

[4] Q. Lindsey, D. Mellinger, and V. Kumar, "Construction of cubic structures with quadrotor teams," in 2011 Robotics: Science and Systems, Los Angeles, CA, Jun. 2011.

[5] M. Manubens, D. Devaurs, L. Ros, and J. Cortés, "Motion planning for 6-D manipulation with aerial towed-cable systems," in 2013 Robotics. Science and Systems, Berlin, Germany, May 2013.

[6] K. Sreenath, N. Michael, and V. Kumar, "Trajectory generation and control of a quadrotor with a cable-suspended load-A differentially-flat hybrid system," 2013, pp. 4888-4895.

[7] G. Gioioso, A. Franchi, G. Salvietti, S. Scheggi, and D. Prattichizzo, "Hand driven UAV formation for cooperative grasping and transportation: the flying hand," in RSS 2013 Work. on Aerial Mobile Manipulation, Berlin, Germany, June 2013.

[8] M. Mohammadi, A. Franchi, D. Barcelli, and D. Prattichizzo, "Cooperative aerial tele-manipulation with haptic feedback," in 2016 IEEE/RSJ Int. Conf. on Intelligent Robots and Systems, Daejeon, South Korea, Oct. 2016, pp. 5092-5098.

[9] H.-N. Nguyen, S. Park, and D. J. Lee, "Aerial tool operation system using quadrotors as rotating thrust generators," in 2015 IEEE/RSJ Int. Conf. on Intelligent Robots and Systems, Hamburg, Germany, Oct. 2015, pp. 1285-1291.

[10] D. Prattichizzo and J. C. Trinkle, "Grasping," in Springer handbook of robotics. Springer, 2008, pp. 671-700. 